\title{
Forschung in der Spannung zwischen Erheben des Status quo und pädagogischer Realisierung einer Vision - aufgezeigt am Beispiel Lebens-, Wert- und Sinnorientierung Jugendlicher im Hinblick auf die Verwirklichung von Inklusion
}

\section{Research in the tension between measuring the educational status quo and a pedagogical realization of a vision - demonstrated by the example of orientation concerning the life, value and sense of adolescents with regard to the implementation of inclusion}

\section{Abstract}

In the article, the current KPH Graz Jugendwertestudie is displayed:

- In the historical development process, as a research from the „Firmungsstudie" to the intercultural and inter-religious Values Study

- Classified in an interdisciplinary question, namely the sense and value of life

- Tolerance was established as a fundamental value regarding the study

- The "more" of "tolerance", namely the "acceptance" from the religions, for example the Islam in terms of the "Projekt Weltethos" (project world ethos) (H. Küng), where all religions shall use their ethical potential for the world's future.

- The learning of empathy and solidarity is viewed in an European dimension and is based on the vision of a worldwide inclusive society and a concretization of "steps into the future" is indicated too. The current European research project ETHOS has investigated the needs of all stakeholders (children, students, teachers, parents) and has developed holistic pedagogical-didactic materials for the age group of 3 to 15 . 
- The inspiring principles of the Catholic Social Doctrine as a major impetus towards a "planetarischen Bewusstseins" (planetary consciousness) (L. Boff) are going to be included, where we see ourselves as "citizens of the world" and can understand and help to form a new, more inclusive society.
\end{abstract}

\title{
Keywords
}

Inclusion, value, sense, youth, empathy, solidarity

Forschung stellt entsprechend ihrer Zielsetzung fest, ,was ist" und ,wie etwas ist'. An einer Pädagogischen Hochschule fragt man sich: Was fängt man mit den Ergebnissen der Forschung an? Inwiefern bringen sie uns weiter? Als einzelne? Als Gesellschaft? Als Menschheit?

Daher sei gleich am Beginn festgehalten: Auch wenn Detailaspekte des Lebens, des beruflichen Feldes usw. erforscht werden: Gegenstand der Forschung ist der Mensch; der Mensch in seinem Wesen, d.h. in seiner Beziehungsfähigkeit.

In dem Artikel wird die aktuelle Jugendwerte-Studie der KPH Graz dargestellt:

- im geschichtlichen Entstehungsprozess,

- eingeordnet in eine interdisziplinäre Fragestellung, nämlich nach Sinn und Wert des Lebens,

- Toleranz als grundlegender Wert von der Studie her begründet,

- das ,Mehr' gegenüber ,Toleranz', nämlich ,Akzeptanz ${ }^{6}$ vom Islam her gesichtet und

- das Erlernen von Empathie und Solidarität wird in einer europäischen Dimension und von der globalen Vision einer inklusiven Gesellschaft angeschaut und eine Konkretisierung in Schritten angedeutet.

\section{Von der „Firmungsstudie“ zur interkulturellen und interreligiösen Wertestudie}

Im Sommersemester 2010 begannen wir, ein ForscherInnenteam an der KPH Graz, ein Forschungsprojekt über ,Firmung in steirischen Pfarren'. Forschung betrieben wir in römisch-katholischen Pfarren, denn die Firmung ist ein Sakrament der katholischen Kirche. Damit war der Fokus auf eine spezielle Zielgruppe gerichtet, und zwar auf die römisch-katholischen Jugendlichen im Alter von 14/15 Jahren; denn in der Steiermark ist das 
Firmungsalter in der Regel zwischen 14 und 15 Jahren. Und auch von dieser Zielgruppe erfassten wir nur solche Jugendlichen, die sich entschieden, sich firmen zu lassen. Dies ist zwar nach wie vor ein sehr großer Teil, aber es gibt auch Jugendliche, die sich nicht firmen lassen. Diese konnten in der Studie nicht erfasst werden, da kein Zugang zu dieser Gruppe von Jugendlichen möglich war. Spannend wären in diesem Zusammenhang drei Fragen: Was sind die Gründe, warum sie sich nicht firmen lassen? Welche, wenn auch (sehr) geringe Beziehung haben solche Jugendlichen zur Institution Kirche? Was sind die Gründe für die Distanz zur Kirche?

Unsere Forschungsfragen bezogen sich auf die Motivation zur Firmung und die Werte dieser römisch-katholischen Jugendlichen, die sich firmen lassen, in den Skalen Hedonismus versus Verzichten-lernen, Religiosität und Glaube, Beziehung zur Kirche ${ }^{1}$, auch im Vergleich mit der großen evangelischen Konfirmandenstudie ${ }^{2}$. Zwar sollte das Projekt Firmung weitergeführt werden, doch erfolgte vom bm:ukk eine Ausschreibung für Forschungsprojekte. In der Vermutung, dass ein Ministerium für Unterricht nicht Gelder für seelsorgliche Anliegen zur Verfügung stellt, erweiterten wir unsere Fragestellung auf den Bereich von Lebens-, Wert- und Sinnorientierung Jugendlicher in der Schule, mit Blick auf den interkulturellen und interreligiösen Kontext, und reichten einen Forschungsantrag ein.

Unser Antrag wurde im November 2010 genehmigt, und zwar für die Dauer von vier Semestern, wobei das Wintersemester 2010 schon als erstes Forschungs-Semester berechnet wurde. Mit dem neuen Forschungsfeld Schule erfassten wir eine Zielgruppe über den römisch-katholischen Bereich hinausgehend, nämlich Jugendliche aller christlichen Konfessionen, z.B. evangelische und orthodoxe. Auch wurden Jugendliche anderer Glaubensrichtungen (v.a. Moslems, Buddhisten ...) erreicht und sogar solche, die sich zu keiner Glaubensgemeinschaft bekennen, also dezidiert ohne religiöses Bekenntnis (o.r.B.) sind. Somit kann die Studie über Lebens-, Wert-

1 R. Straßegger-Einfalt,Firmlinge-WasjungeMenschen denken! Einegeschlechtsspezifische Betrachtung einer Befragung von Firmlingen über ihre Motive zur Firmung über ihre Beurteilung von Sinngenuss, Religiosität und Kirche und ihre Wertehierarchie, in: I. Benischek u.a. (Hrsg.), Empirische Forschung zu schulischen Handlungsfeldern. Ergebnisse der ARGE Bildungsforschung an Pädagogischen Hochschulen in Österreich. Band 2, Wien - Münster 2012, 341-359, hier: 246f.

2 F. Schweitzer, W. Ilg, H. Simojoki (Hrsg.), Confirmation work in Europe - an international comparative study, Gütersloh, Gütersloher Verlagshaus 2010. 
und Sinnorientierung Jugendlicher als repräsentativ angesehen werden, da sie Jugendliche mit verschiedenen (religiösen) Einstellungen erfasst. Erreicht wurden auch Jugendliche verschiedener Kulturen, da v.a. seit der politischen Wende 1989/90 viele Menschen anderer Kulturen aus Ex-Jugoslawien nach Österreich kamen und sesshaft wurden.

Unsere Forschungsfrage lautete: ,Wie nehmen Jugendliche Inklusion bzw. Integration wahr? Wie weit spiegeln sich Inhalte der Salamanca-Erklärung in dieser Altersgruppe unserer Gesellschaft wider? Inwiefern nehmen Jugendliche Elemente dieses Leitprinzips überhaupt wahr und welche Optionen ergeben sich daraus für eine nachhaltige Realisierung dessen in der österreichischen Gesellschaft?‘

Wesentlich war für uns die Frage, wie die Jugendlichen Orientierung im Leben finden und ihrem Leben Wert und Sinn geben.

\section{Was macht mein Leben sinn- und wertvoll?}

Die Frage nach Sinn und Wert des Lebens ist eine der wesentlichen Fragen, die entscheidend unsere Existenz betreffen. Letztlich muss darauf jede/r selbst seine/ihre Antwort finden. Besonders Jugendliche stellen sich diese Frage in sie existentiell betreffender Weise. $\mathrm{Zu}$ den vielen Studien in Bezug auf die Wertewelt Jugendlicher noch eine zusätzliche Studie machen?

Uns interessierte interdisziplinär auch der pädagogische und religionspädagogische Gesichtspunkt, mit der relativ jungen Fragestellung der Multikulturalität und Multireligiosität. Denn erst seit 1994 wird von der UNESCO, der Welterziehungsorganisation, der Blick auf eine, inclusive society` gerichtet. Dabei geht es um die Verwirklichung des Leitprinzips, das besagt, „dass Schulen alle Kinder, unabhängig von ihren physischen, intellektuellen, sozialen, emotionalen, sprachlichen oder anderen Fähigkeiten aufnehmen sollen. Das soll behinderte und begabte Kinder einschließen, Kinder von entlegenen und nomadischen Völkern, von sprachlichen, kulturellen und ethnischen Minoritäten sowie Kinder von anders benachteiligten Randgruppen und -gebieten“3.

3 UNESCO: The Salamanca Statement and Framework for Action on Special Needs Education. Adopted by the World Conference on Special Needs Education: Access and Quality, Salamanca, 
Aber blicken wir zunächst auf Antworten, welche die verschiedenen Wissenschaften auf die Frage nach dem Sinn des Lebens geben. Jede Wissenschaft deutet, d.h. interpretiert, und geht von einem bestimmten Bild vom Menschen aus. Dieses sollte stets offengelegt werden.

Die Verhaltensforschung fand durch Charles Darwin eine Antwort, das Leben sei ein ,struggle for life', ein ,struggle for existence', ein Kampf ums Dasein, bei dem nur der Stärkere überlebt (,survival of the fittest $\left.{ }^{\natural}\right)^{4}$. Es ist eine biologische Tatsache in der Natur, dass die gesünderen und kräftigeren Lebewesen überleben, schwächere Tiere hingegen sterben.

Was Darwin in Bezug auf das Verhalten der Tiere erforscht hat, hat seinen unangefochtenen Wert; aber lässt sich damit schon eine Schlussfolgerung auf den Menschen und vor allem einen angeborenen Egoismus ziehen - ist dieses Bild vom Leben wirklich haltbar?

Charles Darwin wird von den sogenannten Neo-Darwinisten einseitig interpretiert. Die Neurowissenschaft aber hat durch neueste Forschungen die These der Neo-Darwinisten, dem Menschen wäre ein sogenanntes ,egoistisches Gen ${ }^{65} \mathrm{zu}$ eigen, massiv in Frage gestellt und widerlegt. Gerald Hüther ${ }^{6}$ und Joachim Bauer betonen, wie sehr der Mensch schon biologisch-genetisch (vgl. die paarig angelegte DNA) auf Beziehung hin angelegt ist; sie legen dar, dass der Mensch genetisch auf Kommunikation, Kooperation und Kreativität hin angelegt ist ${ }^{7}$. Wert und damit Sinn liegt somit - neurologisch begründet - in der Beziehung, Begegnung, in der Kommunikation im ursprünglichen Sinn (communio facere, lat.) in Richtung ,mehr Gemeinschaft'.

Selbst Medizin und Biologie belegen neuerdings, wie sehr der Mensch ein ,Wesen der Kommunikation“ ist, normalerweise durch körperliche

Spain, 7-10 June 1994, http://www.unesco.org/education/pdf/SALAMA_E.PDF (08.07.2011).

$4 \mathrm{Ch}$. Darwin, On the origin of species by means of natural selection, or the preservation of favoured races in the struggle for life, http://caliban.mpiz-koeln.mpg.de/darwin/origin/chapter3. html (02.08.2012).

5 R. Dawkins, Das egoistische Gen, Reinbeck bei Hamburg 2004.

${ }^{6}$ G. Hüther, Wie lernen Kinder? Voraussetzungen für gelingende Bildungsprozesse aus neurobiologischer Sicht, in: Caspary Ralf (Hrsg.), Lernen und Gehirn. Der Weg zu einer neuen Pädagogik, Freiburg 2006, S. 70-84.

7 J. Bauer, Das kooperative Gen - Abschied vom Darwinismus, Hamburg, Hoffmann und Campe 2009. 
Kommunikation zweier Wesen entstanden, und seit der täglichen Zellteilung zwischen Fötus / Embryo und Mutter ständig kommunizierend ${ }^{8}$. Schon im Uterus lernt der Mensch zu kommunizieren.

Daher greift die Anthropologie zu kurz, wenn sie den Menschen zu sehr als Einzelwesen betrachtet und zu wenig in seiner Beziehungshaftigkeit und Beziehungsfähigkeit in den Blick nimmt. Bereits vom Terminus, anthropos ${ }^{6}$ (äv $\theta \rho \circ \pi \circ \varsigma$, gr.) ist der Mensch einer, der fähig ist, über sich hinauszuschauen, er ist der ,Aufblickende', d.h. ein Wesen mit der Fähigkeit, sich selbst zu übersteigen. Er ist das Wesen, das nicht egozentrisch oder gar egoistisch in sich fixiert sein muss, sondern fähig ist, Beziehung zu anderen aufzunehmen; er ist fähig, zu hören, was ein anderer sagt, und zu schauen, wer zu ihm spricht. Er ist fähig, einen anderen anzusehen und ihm damit Ansehen zu verleihen.

Die Bibel sieht vom ersten Buch an den Menschen als Beziehungswesen, als Mann und Frau (biblische Anthropologie). Gen 1,26 und 27 sprechen davon, dass Gott den Menschen als Mann und Frau erschaffen hat. Mit dem kleinen Wörtchen ,und' ist auch ausgesagt, was ,Gottebenbildlichkeit‘ meint, nämlich die Fähigkeit zu kommunizieren. Der christliche Glaube beschreibt Gott als Vater, Sohn und Heiligen Geist, d.h. als Wesen, die ineinander in Beziehung sind und Beziehung zum Menschen wollen. $\mathrm{Zu}$ seiner wahren Gestalt findet der Mensch also nicht in seiner Ich-Verhaftetheit als Einzelwesen, sondern in Kommunikation mit anderen. Ferdinand Ebner ${ }^{9}$ und Martin Buber ${ }^{10}$ haben in ihren Philosophien zum Ausdruck gebracht, dass der Mensch nur „am Du zum Ich“ wird und damit das dialogische Prinzip des Lebens verwirklicht.

Es stellt sich also die Frage nach dem Wert des Lebens in einer besonderen Weise, wobei der Begriff, Wert' ein sehr junger Begriff ist, der in der Wertphilosophie im 19. Jh. unter wirtschaftlichem Aspekt kreiert wurde: Was etwas ,wert` ist, lässt sich pekuniär in Zahlen, in Euro oder Dollar ausdrücken.

8 G. Dohr, Was istein Embryo?, in: Esterbauer Reinhold(Hrsg.), Wort Wechsel. Sprachprobleme in den Wissenschaften interdisziplinär auf den Punkt gebracht, Würzburg, S. 157-165; M. Beck, Leben - Wie geht das? Die Bedeutung der spirituellen Dimension an den Wendepunkten des Lebens. Wien-Graz-Klagenfurt 2012.

9 F. Ebner, Das Wort und die geistigen Realitäten - Pneumatologische Fragmente, Wien 1952 (Erstveröffentlichung 1921).

${ }^{10}$ M. Buber, Ich und Du, Stuttgart 2008 (Erstveröffentlichung 1922). 
Je höher das Honorar (die Ehre für erbrachte Leistung) ist, umso größer ist der von der Gesellschaft eingeschätzte Wert einer Arbeit.

Daher verwenden manche theologische Ethiker anstatt des Wertbegriffs lieber den ursprünglichen Terminus ,Gut' (,bonum', lat.; vgl. Thomas von Aquin) und fragen: Was ist das Gute im Leben? Inwiefern ist das Leben das fundamentale Gut und die Basis, das Grundlegende für alle anderen Werte, die darauf aufbauen? Denn die Werte Eigentum, Freiheit etc. lassen sich nur verwirklichen, wenn das Leben gegeben ist. Es ist daher ,Aufgabe', aus dem Gegebenen des Lebens etwas zu machen, das gut uns und wertvoll ist. Denn das Ziel theologischer Ethik das gelingende Leben, das Glücken des Lebens ist.

Wie kann dieses gelingende Leben verwirklicht werden? Die Humanwissenschaften, also die Wissenschaften von der Humanität des Lebens, wollen behilflich sein, z.B. die Psychologie, die die Entwicklung, das Lernen, das Verhalten und das Innere des Menschen erforscht. Sie sieht, dass der Mensch nicht nur aus Knochen, Zellen, Muskeln, Wasser, Adern etc. besteht, sondern dass dahinter mehr steckt. Motivationen, Emotionen u.v.a.m. werden wahrgenommen im Hinblick darauf, den Menschen als ,beseeltes' Wesen zu sehen und die Seele als ,das verbindende Urprinzip“, als ,Antreiberin zum Leben“, als ,Kontaktorgan“ zwischen Ich und Du, als ,Quelle der Liebe', als ,Verbindung auch mit den Verstorbenen ${ }^{611}$. Vor 100 Jahren wurde von Carl Gustav Jung und Sigmund Freud wahrgenommen, was noch tiefer steckt als das Sicht- und Beschreibbare, wobei dieses nur die Spitze (d.h. ein Zehntel) eines Eisberges wäre, die anderen neun Zehntel kämen nur in Träumen zum Vorschein und lägen im Verdrängten, im Vergessenen, d.h. im Dunkel des Bewusstseins.

Die Soziologie beschreibt den Menschen in seiner Gefährtenschaft (socius, lat. $=$ der Gefährte) in verschiedenen Lebensräumen und Gemeinschaften, stets im Blick auf den Fokus: Was macht das Humanum aus? In verschiedenen Disziplinen wird der Mensch nicht in erster Linie als Einzel-

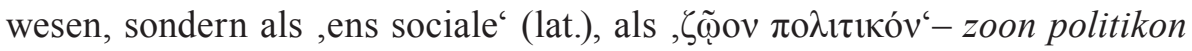
(gr.) beschrieben.

Eine entscheidende Frage der Zukunft wird sein, ob und wie es uns gelingen wird, egoistische Tendenzen zu reduzieren, der Brüchigkeit und Fragmentarität des Lebens im Kleinen und Großen entgegenzusteuern, sowohl im mikro- als

\footnotetext{
11 A. Grün, W. Müller, Was ist die Seele: Mein Geheimnis - meine Stärke, München 2008.
} 
auch im makrosozialen Bereich besser zu kommunizieren und ,Menschen der einen Erde' zu werden, mit einem ,planetarischen Bewusstsein' und auch einer, planetarischen Spiritualität $t^{\prime 2}$.

Unsere Studie will den Menschen als Beziehungswesen in den Blick nehmen, fundiert auf der biblischen Anthropologie, d.h. mit der Frage: Was denkt, fühlt und erlebt der/die Jugendliche auf der Suche nach der Sinnhaftigkeit des Lebens? ${ }^{13}$.

\section{Toleranz - ein grundlegender Wert für das Zusammenleben}

Humanität, Solidarität, Toleranz, Friede, Gerechtigkeit, Gleichberechtigung und Umweltbewusstsein sind handlungsleitende Werte unserer demokratischen Gesellschaft und unseres Rechtsstaates und als solche als allgemeine Bildungsziele in den Lehrplänen verschiedener Schultypen festgeschrieben.

\subsection{Was wurde untersucht?}

In einem Teilprojekt unserer Studie wurde der Frage, welche Faktoren die Einstellung zu Toleranz beeinflussen, nachgegangen, um Ansatzpunkte für eine wirksame Intervention identifizieren zu können. Das erkenntnisleitende Interesse lag im Verstehen und in der Erklärung des Zustandekommens eines so wichtigen Bildungszieles wie Toleranz. Es stellte sich die Frage, ob die Einstellung zur Toleranz eher aus dem Individuum und/oder den Sozietäten (z.B. Klassen, Schulen, Freundesgruppen), in dem sich das Individuum (z.B. Klasse, Freundesgruppe etc.) bewegt, zu verstehen ist.

Folgendes Modell wurde mittels einer Befragung $(n=2361)$ an allgemeinbildenden höheren Schulen, Berufsschulen und landwirtschaftlichen Schulen in der Steiermark überprüft:

${ }^{12}$ L. Boff, Aus dem Scheitern Kraft gewinnen. Auf dem Weg zu einer neuen Spiritualität, in: H. Rothbucher u.a. (Hrsg.), Erfolg und Scheitern. Warum entwickeln sich Kinder nicht so, wie ErzieherInnen es wollen?, Salzburg 1996, Otto Müller, S. 30-42.

${ }^{13}$ F. Feiner, Was macht mein Leben sinn- und wertvoll?, in: F. Feiner, G. Pickl, H. Schwetz, R. Straßegger-Einfalt, U. Straßegger \& B. Swoboda (Hrsg.), Jugend in ihrer Vielfalt-ihre Werte, ihre Perspektiven. Ergebnisse einer Studie der KPH Graz zu Erlebens- und Befindenswelten Jugendlicher, Graz 2013, S. 9-12. 


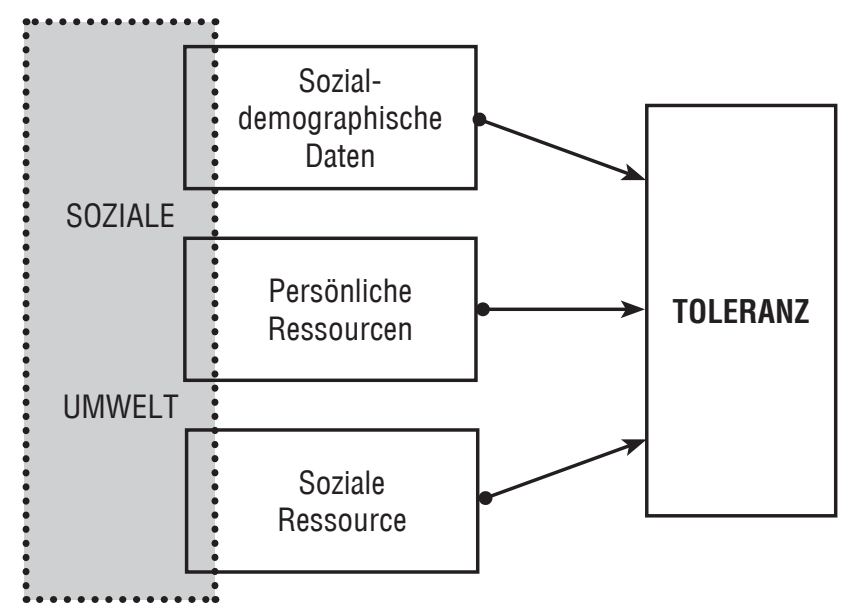

Abb 1: Schwetz / Sever-Wilfinger / Straßegger-Einfalt: Toleranz

Die Dimension Toleranz wurde mit folgenden Items erhoben. Der Wortlaut der Aussagen war:

,Wichtig ist mir ...

... Respekt gegenüber Menschen mit einer anderen Religion;

... andere achten;

... tolerant sein;

... Respekt gegenüber Menschen mit einer anderen Ansichten;

... anderen helfen;

... tolerant sein gegenüber Menschen aus nicht-europäischen Ländern und Kontinenten (z.B. Afrika, Tschetschenien usw.);

... Respekt gegenüber Bettlern aus Hostice (Slowakei)؛

\subsection{Die Ergebnisse}

Bezugnehmend auf das dieser Studie zugrunde gelegte Modell kann festgestellt werden, dass Prädiktoren (bzw. Kovariate) aus allen drei Bereichen einen signifikanten Einfluss auf die abhängige Variable haben. Ein Kontexteinfluss (Klassenzugehörigkeit) konnte nicht nachgewiesen werden.

Generell lässt sich sagen, dass folgende Variable aus dem Bereich der persönlichen Ressourcen (Selbsteinschätzung) einen signifikanten Einfluss haben:

(1) Erlernen von Sprachen und Kennenlernen anderer Länder und Kulturen;

(2) Bereitschaft, Neues auszuprobieren;

(3) Einschätzung der Bedeutsamkeit des Lesens; 
(4) Optimismus;

(5) Bedeutsamkeit guter Umgangsformen und guter Manieren;

(6) Gläubigkeit;

(7) Interesse an österreichischer Politik und

(8) Interesse an internationaler Politik

Weiters kann festgestellt werden, dass folgende Variablen aus dem Bereich der sozialen Ressourcen einen signifikanten Einfluss haben:

(1) Einschätzung der Bedeutung des Freundeskreises und

(2) Einschätzung der Bedeutung dessen, was Vater und Mutter sagen.

Beim Versuch, die Liste der wichtigen Faktoren zusammen zu fassen, kann der Schluss gewagt werden, dass optimistische, an Politik, Sprachen und Kulturen interessierte und dem Neuen gegenüber aufgeschlossene Jugendliche eine positive Einstellung gegenüber Toleranz haben. Diesen Jugendlichen ist auch der Freundeskreis sehr wichtig und sie sind offen für den Rat der Eltern ${ }^{14}$.

\section{Weitere Perspektiven}

Mit dem Titel ,weitere' Perspektiven ist Mehreres gemeint; einerseits im Sinne von ,weiterführend', aber auch aus einer gewissen ,Begrenztheit" in eine größere, Weite' führend.

\subsection{Akzeptanz ist mehr als Toleranz}

Angesichts der enormen Probleme in der Welt entwickelte Hans Küng die Vision eines globalen Bewusstseinswandels, denn die Menschen - weltweit, national und lokal - sind für ein friedliches Zusammenleben auf gemeinsame elementare ethische Werte, Maßstäbe und Haltungen angewiesen. Solche Werte finden sich in allen großen religiösen und philosophischen Traditionen der Menschheit. Nötig sind ein Dialog der Religionen und Kulturen und eine kulturübergreifende Werteerziehung. Inspiriert vom ,Projekt Weltethos ${ }^{15}$ verabschiedete 1993 das Parlament der Weltreligionen in Chicago die

${ }^{14}$ H. Schwetz, Ch. Sever-Wilfinger, R. Straßegger-Einfalt, Toleranz, ein wichtiger Baustein unserer Gesellschaft aus der Perspektive von 14- bis 20-jährigen Jugendlichen, in: I. Benischek u.a. (Hrsg.), a.a.O. 361.

${ }^{15}$ H. Küng, Projekt Weltethos, München 1990. 
,Erklärung zum Weltethos ${ }^{{ }^{16}}$ mit den Kernelementen ,Prinzip Menschlichkeit und ,Verpflichtung auf Gewaltlosigkeit, Gerechtigkeit, Wahrhaftigkeit und die Partnerschaft von Mann und Frau'.

Gesehen werden muss, dass Konflikte nicht in den Religionen, sondern in unterschiedlichen Kulturen wurzeln und durch die Politik oft verfestigt werden. Alle Religionen haben hohe Ideale, deren Verwirklichung oft nur einem begrenzten Maß gelingt. Am Beispiel ,Islam‘ sei exemplarisch aufgezeigt, wie der Wert ,Akzeptanz' von der Religion her eingebracht werden kann.

Einige grundlegende Werte im Islam sind das Sich-Anstrengen - ,Sa'y', Einander-Helfen - ,Muaawanah“, Seine-Zunge-vor-schlechten-Worten-Bewahren - ,Hifsu 1-Lisaan', Versprechen-und-Wort-Halten - ,Wa'd-'Ahd', Genügsamkeit - ,Quanaa'ah' und Vertrauen auf Allah. Neben diesen und vielen anderen islamischen Werten spielen auch Toleranz und Akzeptanz als Werte und gleichzeitig als Verhaltensmuster im Islam und in der Tradition eine wichtige Rolle.

Die UNESCO definiert: „Toleranz bedeutet Respekt, Akzeptanz und Anerkennung der Kulturen unserer Welt, unserer Ausdrucksformen und Gestaltungsweisen unseres Menschseins in all ihrem Reichtum und ihrer Vielfalt. Gefördert wird sie durch Wissen, Offenheit, Kommunikation und durch Freiheit des Denkens, der Gewissensentscheidung und des Glaubens“"17.

Diese Definition der UNESCO hat generell auch im Islam seine Gültigkeit, wobei hier noch einmal zwischen Toleranz und Akzeptanz differenziert wird. Im Islam geht der Begriff ,Akzeptanz ${ }^{‘}$ noch über das oben Genannte hinaus, indem er das ,Andere“ nicht nur respektiert, sondern als gleichwertig anerkennt.

Akzeptanz wurde im Sinne der Wortbedeutung von Muhammad verbreitet, um Frieden und Gemeinschaft zu gewährleisten. Dies zeigt sich in der Charta von Madina, die das Zusammenleben zwischen den Bewohnern Madinas unabhängig von Unterscheidungsmerkmalen wie Ethnie, Abstammung, Religion, Hautfarbe, Sprache, Geschlecht etc. regelt. Diese Charta zeigt, was Akzeptanz im Islam bedeutet. Alle Bewohner Madinas mit

16 Parlament der Weltreligionen, Die Weltethos-Erklärung, in: http://www.weltethos.org/datage/c-10-stiftung/13-deklaration.php (30.12.2012).

${ }^{17}$ Deutsche UNESCO-Kommission e.V., Erklärung von Prinzipien der Toleranz, http://unesco. de/erklaerung_toleranz.html (04.12.2012). 
ihren unterschiedlichen Interessen werden in der Charta nicht nur toleriert, sondern als gleichwertig betrachtet; ihnen werden jeweils die gleichen Rechte zugestanden und die gleichen Pflichten auferlegt ${ }^{18}$.

Religion: Eine Grundlage des Islam ist es, andere Religionen vollständig zu respektieren und zu akzeptieren. Im Qur'an (Sure (22:40) und in Aussagen Muhammads wird offensichtlich, dass im Islam Angehörige anderer Religionen akzeptiert werden, wie sie sind.

Nationalität: Die Nationalität spielt im Islam keine Rolle; wichtig ist ausschließlich der Mensch als solcher (Qur'an Sure 49:13). Im Islam wurden die Grenzen zwischen den Ländern aufgehoben, damit der Mensch in einer globalen Gesellschaft leben kann.

Hautfarbe: Die Hautfarbe wurde dem Menschen von Gott gegeben; der Mensch selbst hat darauf keinen Einfluss. Daher sieht der Islam vor, dass Menschen einander nicht anhand ihrer Hautfarben beurteilen, da sie vor Gott gleichwertig sind, und sich daher gegenseitig akzeptieren sollten, unabhängig von ihren Hautfarben oder ihren Nationalitäten.

Geschlecht: Beim Thema ,Geschlecht ${ }^{`}$ schuf der Islam die ersten Voraussetzungen für Akzeptanz, nämlich Anderen gegenüber keine Vorurteile zu haben. Laut Qur'an und Sunna sind Mann und Frau gleichwertig. Sie begegnen einander mit Respekt und akzeptieren einander, wie sie sind, ohne $\mathrm{zu}$ versuchen, einander zu verändern. Von außen werden oft Unterschiede zwischen Frauen und Männern (Kleidungsvorschriften, alltägliche Pflichten...) gesehen; aus religiös muslimischer Perspektive stellen diese Unterschiede jedoch nicht die Gleichwertigkeit der Geschlechter in Frage.

Werte und Verhaltensregeln: Akzeptanz als Verhaltensregel ist in der persönlichen und gesellschaftlichen Dimension von Bedeutung. Um Parallelgesellschaften zu vermeiden und die Entwicklung von Gemeinschaft zu fördern, ist jedoch Akzeptanz von noch größerer Bedeutung als Toleranz. ,Andersartigkeit‘ zu akzeptieren stellt aktuell eine große Herausforderung für jede Gesellschaft dar, insbesondere aber für Jugendliche. Da das Jugendalter generell von Veränderungen und daher oft von Unsicherheit geprägt ist, ist es für Jugendliche mitunter besonders schwierig, das ,Andere` zu akzeptieren, da es als Bedrohung für das sich eben erst entwickelnde Eigene wahrgenommen werden kann.

${ }^{18}$ A. Zaidan, Die Charta (Verfassung) von Medina, in: http://www.enfal.de/charta.htm (04.12.2012). 
Im Großen und Ganzen ist seitens der muslimischen Jugend in Österreich die Akzeptanz für die Umwelt groß, stößt jedoch auf Grenzen, wenn die Jugendlichen selbst Kritik erfahren, vor allem auf persönlicher und religiöser Ebene. Oft ist davon die Rede, was unsere Gesellschaft an Muslimen und Musliminnen stört; wo aber liegen die Grenzen für Akzeptanz bei muslimischen Jugendlichen?

Da die Familie im Islam eine zentrale Rolle spielt, ist es für muslimische Jugendliche generell schwer zu akzeptieren, wenn sie Streitigkeiten oder Beleidigungen innerhalb von Familien beobachten. Diese können von ihnen meist nur toleriert, jedoch aufgrund ihrer eigenen Werte kaum akzeptiert werden. Des Weiteren endet ihre Akzeptanz dann, wenn ihre Religion in irgendeiner Weise beleidigt wird.

Im Allgemeinen ist es jedoch verständlich, dass die Möglichkeiten, Toleranz und Akzeptanz zu zeigen, nicht nur durch die Religion festgelegt sind, sondern sich von Individuum $\mathrm{zu}$ Individuum unterscheiden. Auch wenn die Beurteilung gewisser Handlungsweisen durch die Religion klar geregelt ist, haben Individuen natürlich persönliche Zugänge, mit diesen Handlungsweisen umzugehen.

Akzeptanz stellt einen wichtigen islamischen Wert dar. Aufgabe der gläubigen Muslime und Musliminnen ist es nicht nur, sich stetig im Sinne des Islam zu bemühen und sich hinsichtlich des eigenen Verhaltens an den Grundsätzen des Islam zu orientieren, sondern auch, andere, mitunter sogar gegensätzliche Verhaltensweisen in der Gesellschaft zu akzeptieren. Nur so kann eine globale Gemeinschaft entstehen, in der das bloße EinanderTolerieren schließlich von wahrer Akzeptanz abgelöst wird ${ }^{19}$.

\subsection{Grenzen in Europa überwinden}

Viele europäische Bildungs-Initiativen in Europa zielen auf die Förderung einer Kultur der Freiwilligkeit und Philanthropie von einem frühen Alter an. Leider wurde - mit fehlendem Blick auf Transzendenz - zu viel Vertrauen in die Institutionen gelegt, als ob diese in der Lage wären, den menschlichen Fortschritt automatisch zu liefern. In Wirklichkeit reichen Institutionen allein nicht aus, denn die ganzheitliche menschliche Entwicklung ist in

${ }^{19}$ B. Asker, Toleranz und Akzeptanz im Islam und in der Tradition. Akzeptanz ist mehr als Toleranz. Werte islamischer Jugendlicher, in: F. Feiner, G. Pickl, H. Schwetz, R. StraßeggerEinfalt, U. Straßegger \& B. Swoboda (Hrsg.), a.a.O., S. 205-210. 
erster Linie eine Berufung, die eine freie Übernahme von Verantwortung in Solidarität einschließt. Darüber hinaus erfordert eine solche Entwicklung eine transzendente Sicht der Person. Ohne Gott wird die Solidarität entweder geleugnet oder heruntergespielt. In diesem Zusammenhang bedeutet die christliche Erziehung in Solidarität auch Überwindung verschiedener Grenzen, vor allem mentaler Grenzen ${ }^{20}$.

Zwar wurden mit der Europäischen Union und ihren Zielsetzungen nationale Grenzen überschritten (Handel, Reisefreiheit, Bildung), doch werden Grenzen innerhalb des Kontinents gesetzt zwischen Ländern, die zur EU dazugehören und solchen, die nicht dazugehören. Diese Länder erleben Einschränkungen im Handeln (Zoll); Menschen dieser Länder sind großen Einschränkungen unterworfen beim Reisen (Visumspflicht) und in der Bildung (keine Teilnahme an den Erasmus- und Leonardo da Vinci-Programmen). Vor allem im Bereich der Bildung werden die von der EU gezogenen Grenzen von ForscherInnen, WissenschaftlerInnen und Lehrenden an Universitäten in Nicht-EU-Ländern als exklusiv und somit auch demütigend empfunden.

Es sind Grenzen zu überwinden, hin zu einem globalen Bewusstsein, Menschen der einen Welt zu sein. Ein Reden von einer Ersten, Zweiten oder Dritten Welt, sollte der Vergangenheit angehören, denn es gibt nur eine Welt mit über sieben Milliarden Menschen und alle haben ihre Würde und sollen ein Recht haben, ein Leben in Würde und Wohlstand zu führen.

\subsection{Vision einer globalen inklusiven Gesellschaft}

Die Vorstellung einer ,inklusiven Gesellschaft ${ }^{\star}$ ist in vollem Gange und ergreift wesentliche Bereiche des menschlichen Lebens, nämlich Politik, Kultur und Bildung. Aber ist diese Idee realisierbar?

\subsubsection{Eine Richtung, der man folgen sollte}

1994 ebnete die Salamanca-Erklärung den Weg, Heterogenität nicht als Hindernis, sondern als Chance zu sehen. Nach dem Leitsatz der UNESCO sollte diese Chance allen auch noch so randständigen Menschen gegeben werden, denn die Marginalisierung der Minderheiten bringt uns nicht weiter.

${ }^{20}$ E. Osewska, Solidarity as Overcoming Boundaries in Europe. Concepts and Prospects for Unity and Education, in: F. Feiner, G. Pick1, H. Schwetz, R. Straßegger-Einfalt, U. Straßegger \& B. Swoboda (Hrsg.), a.a.O., S. 169-176. 
Das Statement ist ein Meilenstein in Richtung Inklusion mit dem Ziel, einen allgemeinen Standard und eine Richtlinie für die allgemeine Pädagogik auszuarbeiten.

Basierend auf dem Salamanca-Statement wurde der Index für Inklusion entwickelt und in englischer Sprache im Jahr 2000 veröffentlicht ${ }^{21}$; derzeit ist er in 40 Sprachen auf allen Kontinenten übersetzt worden. Es ist ein bahnbrechendes Werk für eine weltweite Pädagogik für eine Menschheit, die alle einschließt und willkommen heißt.

Der Index enthält ,eine detaillierte Analyse, wie Lernbarrieren reduziert und überwunden werden können und wie die Teilnahme aller Schüler gewährleistet werden kann'. Es ist ein ,Werkzeug', um ein inklusives Leitbild zu entwickeln ${ }^{22}$.

Ursprünglich wurde der Index für Inklusion für ein Kind bestimmt: Wo erlebt dieses Kind Barrieren und wie können diese abgebaut werden? Inzwischen ist der Index für eine ganze Gruppe, Klasse oder Schule erweitert worden. Jüngst wurde er sogar in kommunalen Gemeinschaften angewendet, zum Beispiel in Wiener Neudorf: Wo gibt es Barrieren für das Zusammenleben in unserer Gemeinde und wie können sie überwunden werden? ${ }^{23}$.

Das Ziel ist, dass inklusives Denken und Handeln so selbstverständlich wird, sodass dafür kein eigener Begriff mehr benötigt wird. - Vielleicht bleibt die Idee der Inklusion eine Vision, aber wir sollten uns dorthin bewegen und sogar noch weiter.

\subsubsection{Ein Instrument zur Umsetzung}

Unter einer Vielzahl von Strategien und Maßnahmen scheint heute ein wirksames Instrument für die Entwicklung hin zu einer inklusiven Gesellschaft entwickelt zu werden. Es gibt eine Hoffnung und einen Handlungsansatz für diejenigen, die einen Schlüssel zur Integration in eine Gesellschaft ohne Ausgrenzung suchen. Dieses Werkzeug ist Open Educational Resources (OER) mit dem Ziel der ,Entwicklung einer universellen pädagogischen Ressource für die ganze Menschheit` (Mulder). Der Vorsitzende der UNESCO-OER

${ }^{21}$ T. Booth, M. Ainscow, Index for inclusion. developing learning and participation in schools, http://www.eenet.org.uk/resources/docs/Index\%20English.pdf (02.07.2013).

${ }^{22}$ I. Boban, A. Hinz, Index für Inklusion. Lernen und Teilhabe in der Schule der Vielfalt entwickeln, Halle-Wittenberg 2003.

${ }^{23}$ Inklusionsprojekt. Was ist eigentlich Inklusion?, http://www.wiener-neudorf.gv.at/system/ web/zustaendigkeit.aspx?menuonr=223350725\&detailonr $=222976353$ (30.12.2012). 
Fred Mulder sieht aber auch realistisch, dass es bis zum Erreichen dieses ehrgeizigen Ziels zwischen 20 bis 30 Jahre dauern wird.

Der Ausgleich der globalen Unterschiede hinsichtlich des Zugangs zu Bildung und digitalen Medien ist eine große Hoffnung im Zuge der Verbreitung von OER. Von dem Zugang zur der Nutzung der OER sollen insbesondere wenig entwickelte Länder profitieren; auch die Europäische Kommission hat in den letzten Jahren verstärkt darauf geachtet, Projekte, die sich direkt oder indirekt mit der Entwicklung und Nutzung frei zugänglicher Lerninhalte beschäftigen, zu finanzieren ${ }^{24}$.

Ist also über Bildung eine Chance zu einer inklusiven Gesellschaft gegeben? Für die Verwirklichung dieser Gesellschaft ist es hilfreich, Prinzipien als Maßstab zu nehmen, die in der katholischen Soziallehre verankert sind.

\subsection{Katholische Soziallehre - Gemeinwohlprinzip}

Die klassischen Prinzipien der katholischen Soziallehre, das Person-, Solidaritäts-, Gemeinwohl- und Subsidiaritätsprinzip, verstehen sich als Bezugspunkte für sein-sollendes Handeln in den jeweiligen gesellschaftlichen Umständen; sie sind also Maßstäbe bei der Suche nach Lösungen.

Diese Prinzipien, die ja nicht ursprünglich im kirchlichen Bereich verankert waren, nimmt auch die EU wahr, besonders die ,Kommission der Bischofskonferenzen der EU', die COMECE, mit Sitz in Brüssel. „Diese Kommission besteht aus Vertretern der Bischofskonferenzen in den Mitgliedstaaten und hat die Aufgabe die Entwicklungen der Europäischen Einstellungen zu 'monitoren', das heißt auf Grund der Richtlinie Prinzipien der Soziallehre der Kirche kritisch zu verfolgen“25.

Das Personprinzip ist grundlegend wichtig, besonders angesichts der Tatsache, dass es Schwache, Gebrechliche, Hilfsbedürftige, solche, die keinen Anwalt haben, gibt. Dabei geht es um die Person in Beziehungen, den Menschen, „,sofern er von Natur aus auf Mit-Sein angelegt und zugleich zu einer höheren Ordnung berufen ist, die die Natur übersteigt und diese zugleich überwindet ${ }^{“ 26}$.

${ }^{24}$ I. Sekret, F. Feiner, The Vision of the Inclusive Society - Is it Possible to Realise?, in: F. Feiner, G. Pickl, H. Schwetz, R. Straßegger-Einfalt, U. Straßegger \& B. Swoboda (Hrsg.), a.a.O., S. 177-183.

${ }^{25}$ A. H. v. Luyn, Das Subsidiaritätsprinzip. Bedeutung und Folgerungen für politische Beziehungen, http://www.iwp.uni-koeln.de/RV-Krise\%20als\%20chance/van\%20Luyn\%20-\%20Da s\%20Subsidiarit\%E4tsprinzip\%20met\%20notenapparaat.pdf (30.12.2012).

${ }^{26}$ Johannes XXIII., Mater et magistra, in: http://www.vatican.va/holy_father/john_xxiii/ency clicals/documents/hf_j-xxiii_enc_15051961_mater_ge.html (31.12.2012). 
„Dieses mit Mit-Sein zum Ausdruck gebrachte Merkmal der Person führt zum Solidaritätsprinzip. Der Andere ist für den Menschen ein wichtiger Faktor seiner Entwicklung. Dabei ist diese Angewiesenheit auf den Anderen nicht nur ein Element der Bedürftigkeit des Menschen - ich habe noch kein Baby gesehen, das sich selbst gewickelt hätte -, sondern auch ein wesentliches Element der sozialen und kulturellen Entfaltung. Der Mensch entwickelt seine Fähigkeiten im Kontakt und in der Begegnung mit ... Anderen ... Weil ich den Anderen brauche, bin ich gefordert, auch für ihn da zu sein, wenn er mich braucht ... In unserem Menschsein und der damit gegebenen Menschenwürde liegt der Grund der gegenseitigen Verpflichtung. Theologisch vertieft können wir es so formulieren: In Jesus Christus ist einer unser aller Bruder geworden, damit wir uns geschwisterlich begegnen. Einer hat unser aller Kreuz getragen, damit wir gemeinsam und solidarisch mit dem Anderen das Kreuz tragen. Einer ist für uns alle auferstanden, damit wir uns gemeinsam zur Auferstehung begleiten. Die Person braucht $\mathrm{zu}$ ihrer Verwirklichung aber nicht nur die einzelnen Anderen, sondern auch die Gemeinschaft mit ihren Einrichtungen als Ganzer. Damit ist eine Verantwortung auch der Gesellschaft und ihren Einrichtungen gegenüber gegeben, wie sie im Gemeinwohlprinzip zum Ausdruck kommt. Strukturen gesellschaftlicher Art bedingen zu einem gewichtigen Teil die Entfaltungsmöglichkeiten des Menschen“"27.

Der Einzelne darf nicht dem Gemeinwohl geopfert werden, daher legt die katholische Soziallehre ein Augenmerk auf das Subsidiaritätsprinzip. Der Einzelne hat Verantwortung dort, wo er Handlungsmöglichkeiten besitzt, zu übernehmen. Hilfe darf also nicht entmündigen, sondern „muss

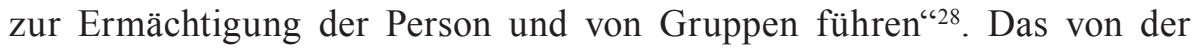
Theologie der Befreiung immer wieder eingeforderte Prinzip der Option für die Armen ist als ein neues Sozialprinzip in die katholische Soziallehre eingeführt worden. Das gesellschaftsgestaltende Potential der Bibel kommt etwa im Wort: ,Was ihr dem Geringsten meiner Brüder und der geringsten meiner Schwestern getan habt, das habt ihr mir getan' (Mt 25,40) zum Ausdruck. „Damit ist gerade für den Christen und die Christin ein Aufruf

${ }^{27}$ L. Neuhold, Wert- und Sinnorientierung in einer Zeit des Wertewandels, in: F. Feiner, G. Pickl, H. Schwetz, R. Straßegger-Einfalt, U. Straßegger \& B. Swoboda (Hrsg.), a.a.O., S. 191-204, hier: 200.

${ }^{28}$ Ebd., S. 201. 
zum Zusammenschluss mit Anderen zum Einsatz für Gestaltung der Gesellschaft ... gegeben“229.

\subsection{Wahrnehmung, Sensibilität, Empathie und Solidarität lernen}

Die Gestaltpädagogik weiß: ,Awareness - Bewusstheit - Wachheit - Gewahrsein' sind Voraussetzungen, wie Lern- und Veränderungsprozesse geschehen können. Und zwar auf beiden Seiten, sowohl der SchülerInnen wie auch der Lehrenden. Es handelt sich um wechselseitige Prozesse, die Lernen, Veränderung und Wachstum ermöglichen. Mit dem gestalttherapeutischen Begriff, awareness" ist nicht Bewusstsein als ,consciousness" gemeint, sondern eher ein ,Gewahrsein ' für das Umfeld und die anderen. Im Grunde geht es um ein umfassendes Wahrnehmen im Sinne des griechischen Begriffes $\alpha i \sigma \theta \dot{\alpha} v \varepsilon \sigma \theta \alpha$ - aisthanesthai, der als Ästhetik ein Bereich der Ethik wurde.

Das Wahrgenommen-werden ermöglicht den Prozess des Sich-selberWahrnehmens und damit des Wahrnehmens möglicher Lösungswege und der notwendigen Ressourcen. Wenn in pädagogischen Beziehungen dem Gegenüber Aufmerksamkeit und Achtsamkeit entgegengebracht werden, entwickelt sich dies auch in ihm, also in einem dialogischen, wechselseitigen Prozess. Sie sind Voraussetzung, damit es zur Produktion und Ausschüttung der körpereigenen Motivationsbotenstoffe wie Dopamin, Opioide und Oxytozin kommt, die für Lebensfreude und positive Lebensenergie verantwortlich sind. Nur wenn einem Menschen Wertschätzung, Interesse und Aufmerksamkeit entgegengebracht werden, kommt es zu diesen Prozessen. Fehlen diese entsteht eine negative Spirale, die sich in Aggression, Lebensunlust und Depression oder im Suchtverhalten seine Wege bahnt.

Das Gehirn zeigt sich also als ,soziales Wesen', das sich in der Interaktion mit dem Umfeld entwickelt, und weniger als allgemein angenommen durch Gene bestimmt ist ${ }^{30}$. Auch für die Neurobiologie gilt, was Martin Buber $^{31}$ das ,dialogische Prinzip nennt: Das Ich wird am Du zum Ich. Die Spiegelneuronen bilden die neuronale Grundlage für unsere Empathie und damit für unsere Beziehungen und Kommunikationsmöglichkeiten. Durch

29 Ebd., S. 201.

${ }^{30}$ G. Hüther, Wie lernen Kinder? Voraussetzungen für gelingende Bildungsprozesse aus neurobiologischer Sicht, in: Caspary Ralf (Hrsg.), Lernen und Gehirn. Der Weg zu einer neuen Pädagogik, Freiburg 2006, S. 70-84.

31 M. Buber, Ich und Du, Stuttgart 2008 (Erstveröffentlichung 1922). 
Spiegelungserfahrungen wird die Empathiefähigkeit gelernt. Fehlen solche Erfahrungen, kann einerseits Autismus entstehen ${ }^{32}$, andererseits kann es eine Wurzel für Gewaltbereitschaft $\operatorname{sein}^{33}$.

Spiegelungserfahrungen sind die Basis für eine Kommunikation, die Schritte in die Zukunft anbahnen hilft.

\subsection{Für eine dialogische, nachhaltige Zukunft - ETHOS}

Ein aktuelles europäisches Forschungsprojekt geht der Frage nach: Wie kann es gelingen, durch ganzheitliches Lernen eine ethische Haltung für eine „dialogische, nachhaltige Zukunft" aufbauen zu helfen?

Die Hauptmotivation für dieses Projekt kommt aus der Erkenntnis, dass die Herausforderungen, denen wir begegnen, nicht rein ökonomischer oder politischer, sondern gleichsam ethischer Natur sind.

Ethos analysierte zunächst den aktuellen Stand der ethischen Erziehung in den teilnehmenden Ländern. Das Ziel ist es, die Bedürfnisse der LehrerInnen / ErzieherInnen (und die der SchülerInnen wie deren Eltern) genau zu definieren. Die ETHOS Bedarfsanalyse identifizierte die Bedürfnisse der Zielgruppen im Feld der ethischen Bildung in der Schule. Insgesamt nahmen 475 Personen an der Befragung teil: 111 LehrerInnen / ErzieherInnen / Stakeholder waren in Fokusgruppen-Interviews involviert, weitere 150 LehrerInnen / ErzieherInnen füllten einen Fragebogen zu ihrer aktuellen Unterrichtspraxis und ihren Bedürfnissen aus, und 214 Eltern/ Erziehungsberechtigte gaben ihre Einschätzungen zur ethischen Bildung ihres Kindes ebenso mittels eines Fragebogens ab. Obwohl die Stichproben in Bezug auf die Herkunftsländer, die Schulformen, das Alter der SchülerInnen und die gelehrten Unterrichtsfächer als sehr heterogen angesehen werden können, zeigte sich ein hoher Konsens aller Beteiligten, sowohl hinsichtlich der hohen Bedeutsamkeit, ethische Werte in der schulischen Bildung zu vermitteln, als auch in Bezug auf die Wahl der relevanten Themen und gewünschten Lehrmethoden. Die Themen, die in allen Ländern und von allen beteiligten Gruppen genannt wurden, sind in der folgenden Graphik gemäß der Häufigkeit ihrer Nennung in unterschiedlichen Größen dargestellt (je häufiger die Nennung eines Wortes, desto größer die Darstellung):

${ }^{32}$ J. Bauer, Warum ich fühle, was du fühlst, Hamburg ${ }^{9} 2006$.

${ }_{33}$ J. Bauer, Schmerzgrenze: Vom Ursprung alltäglicher und globaler Gewalt, München 2011. 
Abb 1: http://www.ethos-education.eu/index.html

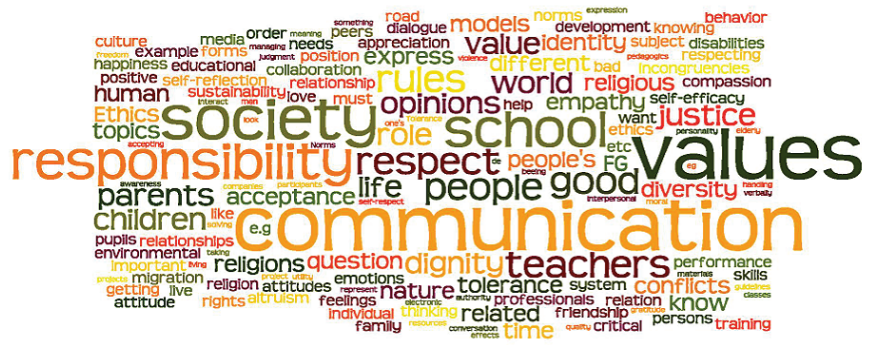

Als Teil der qualitativen Inhaltsanalyse wurden alle qualitativen Fragen (aus Fokusgruppen-Interviews sowie Fragebögen der LehrerInnen / ErzieherInnen und Eltern / Erziehungsberechtigten), die sich inhaltlich mit „relevanten Themen für eine ethische Bildung" beschäftigten, evaluiert. Es zeigte sich, dass LehrerInnen / ErzieherInnen und Eltern/Erziehungsberichtigte in allen befragten Ländern ähnliche Bedürfnisse und Erwartungen haben in Bezug auf:

a) welche Themen sie als besonders relevant für die ethische Bildung ansehen, und

b) zu welchen thematischen Aspekten das ETHOS-Team Unterrichtsmaterialien und Werkzeuge entwickeln und bereitstellen soll.

Die Antworten können in acht Hauptthemen gefasst werden:

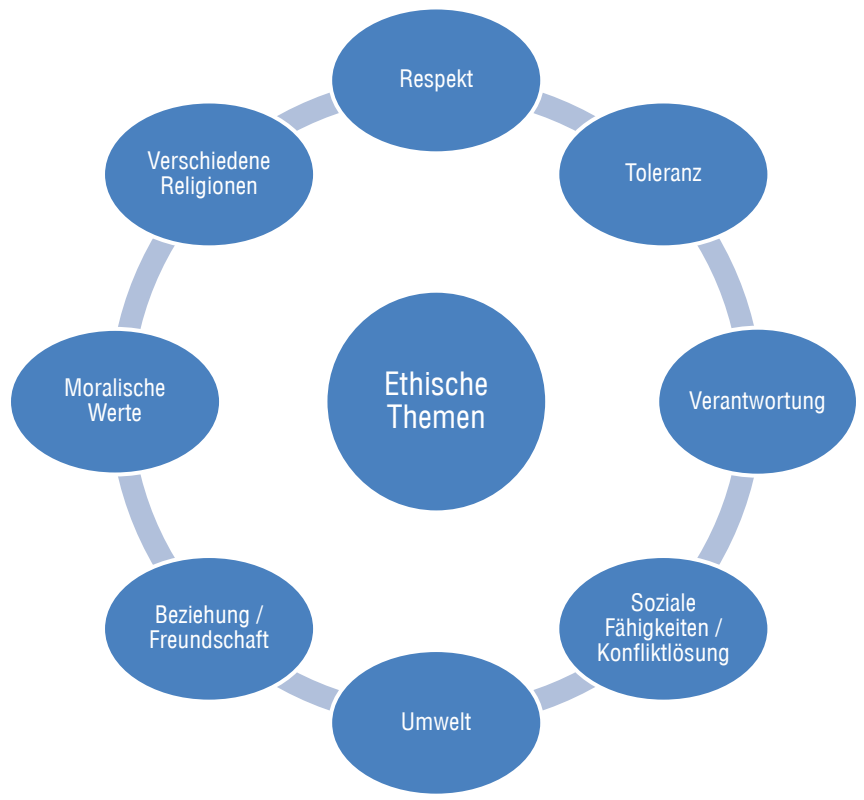

Abb 2: Bevorzugte ethische Themen, die in der ETHOS Erhebung der Benutzer-Bedürfnisse (User Needs Analysis) durch LehrerInnen, ErzieherInnen und Eltern erhoben wurden 
Derzeit werden erzieherische Methoden und Werkzeuge entwickelt, die für Kinder in unterschiedlichen Altersstufen und für LehrerInnen, die sich damit auseinandersetzen wollen.

Die Vorstellung von Ethos ist, ein Referenznetzwerk zu ethischer Bildung zu schaffen, um professionelle pädagogisch-didaktische Methoden für eine Unterrichtspraxis im Hinblick auf nachhaltige und dialogische Zukunft bereitzustellen. Ein Hauptziel ist, eine dialogische und empathische Haltung auf allen Ebenen des Bildungsprozesses zu stärken. Die dialogische Natur der Ethik und der ethischen Erziehung verschreibt sich einer Offenheit gegenüber dem Anderen und eröffnet somit einen Prozess des gegenseitigen Wachstums und Lernens.

Das ETHOS-Team entwickelt altersgerechte pädagogische Materialien und Hilfsmittel für verschiedene Altersgruppen, da es alterstypische Unterschiede in der jeweiligen Stufe der moralischen und emotionalen Entwicklung, in den Bedürfnissen der Kindern, dem Vokabular, dem Erkenntnisstand und der psychischen Entwicklung gibt. Die ETHOSMethoden suchen den unterschiedlichen entwicklungspsychologischen Gegebenheiten gerecht zu werden.

Die pädagogischen Materialien und Hilfsmittel für die ethische Bildung, als auch unser methodisch-didaktischer Ansatz sind sehr spezifisch. Das Trainingsprogramm bietet erstmalig und in höchst innovativer Weise ganzheitliches ethisches Lernen, indem es das Philosophieren ethischer Probleme an Hand altersgerechter Geschichten / Cartoons mit den multiplen Intelligenzen Howard Gardners verknüpft. Mit den Trainingskursen wollen wir LehrerInnen bei der ethischen Bildung unterstützen.

Die Homepage http://www.ethos-education.eu/ bietet eine Fülle von Lehrmitteln für verschiedene Altersgruppen von 3-5, 5-7, 7-9, 9-11 und 1115 in sieben Sprachen an. Erfreulich ist, dass weitere ,associated partner“ in das Programm integrieren, und in Griechenland, der Slowakei, Polen und der Ukraine Forschung betreiben und pädagogisch-didaktische Hilfsmittel sich in ihre Sprachen übersetzen. So entsteht in der Tat ein „Referenznetzwerk ethischer Bildung" die immer mehr europäische Länder umfasst und sogar darüber hinausgeht. 


\section{Schritte in die Zukunft}

Wenn eine inklusive Gesellschaft Wirklichkeit werden soll, muss zuerst eine Vorstellung von einem Weg bestehen. Wir sehen grundgelegte und zu lernende Schritte:

- Ich bin ein Wesen, das durch intensive Kommunikation entstanden ist:Zwei Menschen sind einander begegnet, haben sich aufeinander eingelassen, haben sich einander geschenkt, sodass sie ,ein Fleisch' (Gen 2,24) wurden.

- Ich bin durch Kommunikation entstanden, ich bin fähig zu kommunizieren, lebe durch Kommunikation und gewinne meine Identität durch Kommunikation, ich werde am Du zum Ich (Martin Buber). Seit der Verschmelzung von Samenzelle und Eizelle ereignet sich Kommunikation. Eine Mutter und ihr ungeborenes Kind kommunizieren ständig miteinander während der Zell-Teilung. Die Fähigkeit zu kommunizieren und Bindungen zwischen Menschen zu entwickeln beginnt im Mutterleib.

- Hören und sprechen lernen sind eine wesentliche Basis dafür, dass eine inklusive Gesellschaft entstehen kann. Unser Ohr ist ca. 18 Wochen nach der Zeugung fertig ausgebildet. Es ist somit das erste Organ das seine Tätigkeit aufnimmt - seit der achten Lebenswoche (im Mutterleib) hören wir und mit 18 Wochen ist unsere Hörfähigkeit vollständig ausgebildet.

- Ich bin in der Regel in eine Familie geboren. Im besten Fall ist die Familie der, sozialen Uterus' (Adolf Portmann), in dem ich mich weiterentwickeln kann; die primäre Sozialisation bringt mir wichtige soziale Erfahrungen. Bereits ein Kind mit zwei Monaten sucht Übereinstimmung und es entwickelt sich bei ihm das Grundgefühl, in einer intuitiven Verbindung mit anderen gleichartigen Wesen $\mathrm{zu}$ stehen, mit ihnen in einer gemeinsamen emotionalen Welt zu leben ... Kleinkinder bemühen sich bereits mit zwei Monaten aktiv um eine gefühlsmäßige Abstimmung bzw. Übereinstimmung mit der Mutter. Im dritten Lebensmonat entwickelt es „ein Gefühl dafür, dass es mit eigenen Lebensäußerungen bei seinen Bezugspersonen Verhaltensänderungen auslösen kann ${ }^{\text {‘34 }}$.

- Wertvoll für ein Kind ist es, Geschwister zu haben und Spielgefährten. An ihnen kann es seine Grenzen abstecken und seine Bereiche als Lebensraum gestalten.

\footnotetext{
${ }^{34}$ J. Bauer, Warum ich fühle, was du fühlst, Hamburg ${ }^{92006, ~ S . ~} 63$.
} 
- Um jemandem gut sein zu wollen und zu können, ist die Erfahrung nötig: ,Jemand war gut zu mir, jemand meint es gut mit mir‘. Winnicott spricht von der Notwendigkeit einer ,good enough mother', die nicht die leibliche Mutter sein muss. Aus dieser Erfahrung, dass das Leben gut ist, kann ich jemandem gut sein, Gutes tun und jemandem ein/e FreundIn sein.

- Wie immer die Erfahrung in der Herkunftsfamilie war, den Wunsch, eine Familie zu gründen, gibt es in allen Untersuchungen über Werte der Jugendlichen: Freundschaft und Familie stehen bei den Werten an oberster Stelle.

- Eine Gruppe Gleichaltiger, eine peer-group ermöglicht mir die Erfahrung, dazu zu gehören, inkludiert zu sein, anerkannt zu sein und damit einen Selbstwert zu entwickeln, wodurch ich auch anderen gegenüber wertschätzend handeln kann.

- In einer Schule, wo Inklusion gelingt, fühlt sich jeder willkommen, wird niemand ausgeschlossen und es wird ein wertschätzender Umgang aller Beteiligten miteinander gepflegt. So ist Schule als Lebensraum ein wesentliches Handlungsfeld, in dem die Vision einer inklusiven Gesellschaft realisiert wird. Damit kommen die personalkommunikativen Fähigkeiten des Lehrers/der Lehrerin in den Blickpunkt, weil Wahrnehmung, Sensibilität, Empathie vor allem in personalen Bezügen gelernt wird. Joachim Bauer spricht vom Eröffnen eines ,Zukunftskorridors ${ }^{635}$ in Visionen von Lehrenden, die ihren SchülerInnen zutrauen, dass sie das Leben schaffen. Dann werden die Motivationsbotenstoffe als Grundvoraussetzung für eine positive Lebenseinstellung im Gehirn ausgeschüttet. So tragen diese als ,VorBilder ${ }^{6}$ entscheidend dazu bei, was aus ihnen werden $\mathrm{kann}^{36}$ und sie damit zu ,Baumeistern ihrer selbst‘ (Maria Montessori) im Sinne einer konstruktivistischen Pädagogik werden können.

- Auch in einem erweiterten Lebensraum von Gemeinde und Pfarre gilt es, Barrieren im Zusammenleben abzubauen und ein Zusammenleben in Vielfalt und Heterogenität zu verwirklichen, wo Menschen sich

${ }^{35}$ J. Bauer, Lob der Schule, Hamburg 2007.

${ }^{36} \mathrm{H}$. Neuhold, Das Leben in Freiheit und solidarischer Verantwortung gestalten - ethische Kompetenz entwickeln, in: F. Feiner, G. Pickl, H. Schwetz, R. Straßegger-Einfalt, U. Straßegger \& B. Swoboda (Hrsg.), a.a.O., S. 211-223. 
wohl fühlen können. Die Umsetzung des ,Index für Inklusion', der ursprünglich für ein einzelnes Kind konzipiert war, dann in Bezug auf Klasse und Schule erweitert wurde, ist auf alle - kommunalen Lebensräume auszuweiten.

- Wie definiere ich meine Identität? Fühle ich mich als ÖsterreicherIn, Deutsche/r, Slowen/e/in, SchweizerIn oder BosnierIn? Freue ich mich darüber, dass unser Kontinent Europa ein Teil der Welt ist, wo Friede mehr und mehr realisiert wird?

- Welche Erfahrung machte Europa vor nur ein wenig mehr als zwei Jahrzehnten? 50 km südlich von Graz gab es Krieg, Granaten schlugen sogar in den Kirchturm von Bad Radkersburg ein. Vor 25 Jahren konnte sich kaum ein Mensch vorstellen, dass die zwei politischen Blöcke sich einander annähern und sich der Eiserne Vorhang in ,nichts` auflöst. Wir waren Augen- und Ohrenzeugen des Falls der Berliner Mauer. Eine Grenze zwischen Menschen, die nur unter Todesgefahr unter Einsatz des eigenen Lebens überschritten werden konnte, zerbröselte. Gorbatschow warnte Honecker: ,Wer zu spät kommt, den straft die Geschichte!' Die Erbauer von Mauern sind nicht die Menschen des neuen Europa, es sind die Brückenbauer, die trennende Mauern niederreißen. Seit Schengen Ende 2007 können wir von Portugal nach Rumänien, von Sizilien nach Norwegen fahren, ohne angehalten zu werden.

- In einer Aufsehen erregenden Rede in Salzburg 1995 sprach der Befreiungstheologe Leonardo Boff über eine Richtung, wohin sich die Menschheit bewegen sollte. Zu Beginn zitierte er den Astronauten John W. Young, der im Jahr 1972 vom Mond aus ergreifende Worte sprach: „Da unten ist die Erde. Dieser blau-weiße, herrliche, strahlende Planet - unsere menschliche Heimat! Hier vom Mond aus halte ich ihn mit der blanken Hand. Aus dieser Perspektive gibt es dort weder Weiße noch Schwarze, gibt es keine Spaltung zwischen Ost und West, gibt es weder Kommunisten noch Kapitalisten, weder Nord noch Süd. Alle bilden wir eine einzige Erde. Wir müssen es lernen, diesen Planeten, von dem wir ja ein Teil und ein Stück sind, zu lieben ${ }^{637}$. - Ein wahrlich inklusiver Gedanke.

${ }^{37}$ Zit. in: L. Boff, Aus dem Scheitern Kraft gewinnen. Auf dem Weg zu einer neuen Spiritualität, in: H. Rothbucher u.a. (Hrsg.), Erfolg und Scheitern. Warum entwickeln sich Kinder nicht so, wie ErzieherInnen es wollen?, Salzburg 1996, Otto Müller, S. 30-42. 
- Bin ich in der Lage, meinen Geist zu erweitern? L. Boff erhebt das Postulat eines neuen planetarischen Bewusstseins: „Das wachsende planetarische Bewusstsein macht uns, die bisher Einwohner dieses oder jenes Landes waren, zu Bürgerinnen und Bürger der einen Erde. Wir bilden eine Schicksalsgemeinschaft. Das menschliche Schicksal ... ist unlösbar verbunden mit dem Schicksal des Planeten und des Kosmos" ${ }^{\text {“38 }}$.

- Nur eine solche Offenheit wird uns helfen, eine ,neue Gesellschaft` zu bilden $^{39}$.

\section{Bibliography}

Asker, B., Toleranz und Akzeptanz im Islam und in der Tradition. Akzeptanz ist mehr als Toleranz. Werte islamischer Jugendlicher, in: F. Feiner, G. Pickl, H. Schwetz, R. Straßegger-Einfalt, U. Straßegger, \& B. Swoboda (Hrsg), Jugend in ihrer Vielfalt - ihre Werte, ihre Perspektiven. Ergebnisse einer Studie der KPH Graz zu Erlebens- und Befindenswelten Jugendlicher, Graz 2013, S. 205-210.

Bauer J., Warum ich fühle, was du fühlst, Hamburg ${ }^{9} 2006$.

Bauer J., Lob der Schule. Hamburg 2007.

Bauer J., Das kooperative Gen - Abschied vom Darwinismus, Hamburg 2009.

Bauer J., Schmerzgrenze: Vom Ursprung alltäglicher und globaler Gewalt, München 2011.

Beck M., Leben - Wie geht das? Die Bedeutung der spirituellen Dimension an den Wendepunkten des Lebens, Wien, Graz - Klagenfurt 2012.

Boban I., Hinz A., Index für Inklusion. Lernen und Teilhabe in der Schule der Vielfalt entwickeln, Halle-Wittenberg 2003.

Boff L., Aus dem Scheitern Kraft gewinnen. Auf dem Weg zu einer neuen Spiritualität, in: H. Rothbucher u.a. (Hrsg.), Erfolg und Scheitern. Warum entwickeln sich Kinder nicht so, wie ErzieherInnen es wollen? Salzburg 1996, S. 30-42.

Booth T., Ainscow M., Index for inclusion. developing learning and participation in schools, http://www.eenet.org.uk/resources/docs/Index\%20English.pdf (02.07.2013).

Buber M., Ich und Du, Stuttgart 2008 (Erstveröffentlichung 1922).

Darwin Ch., On the origin of species by means of natural selection, or the preservation of favoured races in the struggle for life, in: http://caliban.mpiz-koeln.mpg.de/darwin/ origin/chapter3.html (02.08.2012).

Dawkins R., Das egoistische Gen, Reinbeck bei Hamburg 2004.

38 L. Boff, Aus dem Scheitern Kraft gewinnen. Auf dem Weg zu einer neuen Spiritualität, in: H. Rothbucher u.a. (Hrsg.), Erfolg und Scheitern. Warum entwickeln sich Kinder nicht so, wie ErzieherInnen es wollen?, Salzburg 1996, Otto Müller, S. 30-42, S. 32.

39 I. Sekret, F. Feiner, The Vision of the Inclusive Society - Is it Possible to Realise?, in: F. Feiner, G. Pick1, H. Schwetz, R. Straßegger-Einfalt, U. Straßegger \& B. Swoboda (Hrsg.), a.a.O., S. 177-183. 
Deutsche UNESCO-Kommission e.V., Erklärung von Prinzipien der Toleranz, http:// unesco.de/erklaerung_toleranz.html (04.12.2012).

Dohr G., Was ist ein Embryo?, in: R. Esterbauer (Hrsg.), Wort Wechsel. Sprachprobleme in den Wissenschaften interdisziplinär auf den Punkt gebracht, Würzburg 2007, S. 157165.

Ebner F., Das Wort und die geistigen Realitäten - Pneumatologische Fragmente, Wien 1952 (Erstveröffentlichung 1921).

ETHOS, http://www.ethos-education.eu/ (18.11.2013).

Feiner F., Was macht mein Leben sinn- und wertvoll?, in: F. Feiner, G. Pick1, H. Schwetz, R. Straßegger-Einfalt, U. Straßegger \& B. Swoboda (Hrsg), Jugend in ihrer Vielfalt - ihre Werte, ihre Perspektiven. Ergebnisse einer Studie der KPH Graz zu Erlebens- und Befindenswelten Jugendlicher, Graz 2013, S. 9-12.

Feiner F., Pickl G., Schwetz H., Straßegger-Einfalt R., Straßegger U. \& Swoboda B. (Hrsg), Jugend in ihrer Vielfalt - ihre Werte, ihre Perspektiven. Ergebnisse einer Studie der KPH Graz zu Erlebens- und Befindenswelten Jugendlicher, Graz 2013.

Gardner H., Frames of mind: The theory of multiple intelligences, New York 1983, Basic Books.

Grün A., Müller W., Was ist die Seele: Mein Geheimnis - meine Stärke, München 2008.

Hüther G., Wie lernen Kinder? Voraussetzungen für gelingende Bildungsprozesse aus neurobiologischer Sicht, in: Caspary, Ralf(Hrsg.), Lernen und Gehirn. Der Weg zu einer neuen Pädagogik, Freiburg 2006, S. 70-84.

Inklusionsprojekt. Was ist eigentlich Inklusion?, http:/www.wiener-neudorf.gv.at/system/ web/zustaendigkeit.aspx? menuonr=223350725\&detailonr $=222976353$ (30.12.2012).

Johannes XXIII., Mater et magistra, in: http://www.vatican.va/holy_father/john_xxiii/ encyclicals/documents/hf_j-xxiii_enc_15051961_mater_ge.html (31.1.12.2012).

Küng H., Projekt Weltethos, München 1990.

Luyn A. H. v., Das Subsidiaritätsprinzip. Bedeutung und Folgerungen für politische Beziehungen, http://www.iwp.uni-koeln.de/RV-Krise\%20als\%20chance/van\%20Luyn $\% 20-\% 20$ Das $\% 20$ Subsidiarit\%E4tsprinzip\%20met\%20notenapparaat.pdf(30.12.2012).

Neuhold H., Das Leben in Freiheit und solidarischer Verantwortung gestalten - ethische Kompetenz entwickeln, in: F. Feiner, G. Pickl, H. Schwetz, R. Straßegger-Einfalt, U. Straßegger \& B. Swoboda (Hrsg), Jugend in ihrer Vielfalt - ihre Werte, ihre Perspektiven. Ergebnisse einer Studie der KPH Graz zu Erlebens- und Befindenswelten Jugendlicher, Graz 2013, S. 211-223.

Neuhold L., Wert- und Sinnorientierung in einer Zeit des Wertewandels, in: F. Feiner, G. Pickl, H. Schwetz, R. Straßegger-Einfalt, U. Straßegger \& B. Swoboda (Hrsg.), Jugend in ihrer Vielfalt - ihre Werte, ihre Perspektiven. Ergebnisse einer Studie der KPH Graz zu Erlebens- und Befindenswelten Jugendlicher, Graz 2013, S. 191-204.

Osewska E., Solidarity as Overcoming Boundaries in Europe. Concepts and Prospects for Unity and Education, in: F. Feiner, G. Pickl, H. Schwetz, R. Straßegger-Einfalt, U. Straßegger \& B. Swoboda (Hrsg.), Jugend in ihrer Vielfalt - ihre Werte, ihre Perspektiven. Ergebnisse einer Studie der KPH Graz zu Erlebens- und Befindenswelten Jugendlicher, Graz 2013, S. 169-176.

Parlament der Weltreligionen: Die Weltethos-Erklärung, in: http://www.weltethos.org/datage/c-10-stiftung/13-deklaration.php (30.12.2012). 
Schlenk E., Meier A.-M, User Needs Analysis and Methodology Development, in: http:// www.ethos-education.eu/resources/D5_User_Needs_Analysis.pdf (18.11.2013).

Schlenk E. unter Mitarbeit von B. Ćurko, F. Feiner, J. Juhant, S. Pokorny, V. Strahovnik, Methodisch-didaktische Empfehlungen. Ein Handbuch für LehrerInnen und ErzieherInnen zur Unterstützung der Entwicklung pädagogische ethische Erziehung und Bildung, in: http://www.ethos-education.eu/resources/D6_Methodology_Guidelines DE.pdf (18.11.2013).

Schweitzer F., Ilg W., Simojoki H. (Hrsg.), Confirmation work in Europe - an international comparative study, Gütersloh 2010.

Schwetz H., Sever-Wilfinger Ch., Straßegger-Einfalt R., Toleranz, ein wichtiger Baustein unserer Gesellschaft aus der Perspektive von 14- bis 20-jährigen Jugendlichen, in: i. Benischek u.a. (Hrsg.), Empirische Forschung zu schulischen Handlungsfeldern. Ergebnisse der ARGE Bildungsforschung an Pädagogischen Hochschulen in Österreich. Band 2, Wien - Münster 2012, S. 361-381.

Sekret I., Feiner F., The Vision of the Inclusive Society - Is it Possible to Realise?, in: F. Feiner, G. Pickl, H. Schwetz, R. Straßegger-Einfalt, U. Straßegger \& B. Swoboda (Hrsg), Jugend in ihrer Vielfalt - ihre Werte, ihre Perspektiven. Ergebnisse einer Studie der KPH Graz zu Erlebens- und Befindenswelten Jugendlicher, Graz 2013, S. 177-183.

Strahovnik V., all partners, D4: Report on existing models of ethical education in schools. Date of Delivery: 1/4/2013.

Straßegger-Einfalt R., Firmlinge - Was junge Menschen denken! Eine geschlechtsspezifische Betrachtung einer Befragung von Firmlingen über ihre Motive zur Firmung über ihre Beurteilung von Sinngenuss, Religiosität und Kirche und ihre Wertehierarchie, in: I. Benischek u.a. (Hrsg.), Empirische Forschung zu schulischen Handlungsfeldern. Ergebnisse der ARGE Bildungsforschung an Pädagogischen Hochschulen in Osterreich. Band 2, Wien - Münster 2012, S. 341-359.

UNESCO, The Salamanca Statement and Framework for Action on Special Needs Education. Adopted by the World Conference on Special Needs Education: Access and Quality, Salamanca, Spain, 7-10 June 1994, http://www.unesco.org/education/pdf/SALAMA_E. PDF (08.07.2011).

Zaidan A., Die Charta (Verfassung) von Medina, http://www.enfal.de/charta.htm (04.12.2012). 